\title{
Right Coronary Artery-Superior Vena Cava Fistula Manifesting as NSTEMI: Case Report, Review of Imaging, and Summary of Guidelines
}

\author{
Perry Wengrofsky ${ }^{1}$, Pramod Theeta Kariyanna ${ }^{2}$, Eric Kupferstein ${ }^{2}$, \\ Howard Levitt ${ }^{3}$, Sudhavna Hegde ${ }^{3}$, Samy I. McFarlane ${ }^{1, "}$ \\ ${ }^{1}$ Department of Internal Medicine, State University of New York, Downstate Medical Center, Brooklyn, N.Y., U.S.A-11203 \\ ${ }^{2}$ Division of Cardiovascular Disease, Department of Internal Medicine, State University of New York, \\ Downstate Medical Center, Brooklyn, N.Y., U.S.A-11203 \\ ${ }^{3}$ Division of Cardiovascular Disease, Department of Internal Medicine, Kings County Hospital Center, Brooklyn, N.Y., U.S.A-11203 \\ *Corresponding author: Samy.mcfarlane@downstate.edu
}

Received March 03, 2019; Revised April 20, 2019; Accepted May 04, 2019

\begin{abstract}
Coronary artery fistulas (CAFs) represent a spectrum of abnormal connections between a coronary artery and another coronary artery, vein, or major blood vessel, known as coronary-vascular fistulas, or between a coronary artery and a cardiac chamber, known as coronary-cameral fistulas. While CAFs generally remain asymptomatic into the fifth decade of adult life, they can present with a diverse symptomatic profile, typically with angina from abnormal myocardial perfusion, or in the setting of larger fistulas, as right- or left-heart failure from pulmonary or left ventricular circulatory overload. CAFs rarely manifest as myocardial infarction in the absence of thrombosis within the fistula. When clinically suspected based on a continuous murmur on physical exam or an accidental finding on radiology, computed tomography angiography (CTA) and coronary angiography are the preferred diagnostic imaging modalities. Fistula anatomic and patient specific characteristics guide clinical decisions on transcatheter or surgical management strategies. We present the case of a right coronary artery-superior vena cava fistula manifesting as a non-ST elevation myocardial infarction. We also present a review of the imaging techniques available for evaluation of CAFs, and a summary of the major national and international cardiology society guidelines on the diagnosis and management of CAFs.
\end{abstract}

Keywords: coronary artery fistula, right coronary artery, superior vena cava

Cite This Article: Perry Wengrofsky, Pramod Theeta Kariyanna, Eric Kupferstein, Howard Levitt, Sudhavna Hegde, and Samy I. McFarlane, "Right Coronary Artery-Superior Vena Cava Fistula Manifesting as NSTEMI: Case Report, Review of Imaging, and Summary of Guidelines." American Journal of Medical Case Reports, vol. 7, no. 5 (2019): 74-78. doi: 10.12691/ajmcr-7-5-1.

\section{Introduction}

Coronary artery fistulas (CAFs) are abnormalities of coronary arterial connection and termination between one or more coronary arteries with another blood vessel, known as coronary-vascular fistulas, or between one or more coronary arteries and a cardiac chamber, known as coronary-cameral fistulas [1]. While predominantly congenital, CAFs can be acquired in the setting of cardiovascular disease such as coronary atherosclerosis and myocardial infarction, or be iatrogenic in origin and occur after procedures such as percutaneous coronary intervention, cardiac implantable electronic device insertion, and endomyocardial biopsy [1,2]. CAFs can involve any combination of coronary arteries, sinuses, and cardiac chambers, and while the overall prevalence of CAFs in the general population is less than $1 \%$, the right coronary artery (RCA) is the most common coronary artery of origin, accounting for $50-60 \%$ of CAFs $[3,4]$. While right sided cardiac chambers and vessels are the most common sites of termination, the superior vena cava (SVC) is an overwhelmingly rare region of termination, representing $1 \%$ of cases $[4,5]$. RCA-SVC fistulas have been previously documented in individual case reports and broader reviews $[6,7,8]$.

Diagnostic evaluation, workup, and management of CAFs is both diverse and difficult, as CAFs can range from being clinically silent to symptomatic with exerciseinduced angina, or be seen as incidental findings on left heart catheterization with coronary angiography or other imaging modalities such as computed tomography angiography (CTA) $[1,9,10]$. Differing pathophysiologic mechanisms are attributed to angina in the setting of CAFs, including 'coronary steal phenomenom' reflecting the abnormal pressure gradient from a high pressure coronary artery to a low pressure venous capacitance chamber, and the clinical significance of specific CAFs that can cause reversible ischemia and angina [1,3]. Myocardial 
infarction (MI), either ST-elevation MI or non-ST elevation MI (NSTEMI), has been previously reported, but is a rare clinical manifestation of CAF in the absence of CAF thrombosis $[1,11,12]$. Given the typical asymptomatic nature and patterns of incidental discovery of CAFs on radiology or angiography, guidelines on diagnostic imaging and management are discrepant and evolving [13,14,15].

We present the case of an NSTEMI as the initial manifestation of an RCA-SVC fistula, accompanied by a review of the imaging modalities for visualization and classification of CAFs, and summary of the major cardiology society guidelines on the diagnosis and management of CAFs.

\section{Case Presentation}

A 57-year-old Jamaican male with a past medical history of diabetes mellitus presented to the emergency department with complaints of syncope after using the restroom. He denied chest pain, palpitations, diaphoresis, and lightheadedness, confusion, tongue biting, and urinary or fecal incontinence. On arrival, patient was afebrile and hemodynamically stable, and physical exam was unremarkable. Electrocardiogram (EKG) on admission demonstrated normal sinus rhythm and minimal ST-segment depressions in leads II and aVF, with early repolarization in leads V2-V3 [Figure 1]. Blood work was significant for elevated troponin $\mathrm{T}$ to $0.136(\mathrm{ng} / \mathrm{mL})$. Chest X-ray revealed lobular perihilar opacities concerning for lymphadenopathy.

He patient underwent thoracic CTA to rule out pulmonary embolism, which was significant for bulky bilateral hilar and mediastinal lymphadenopathy with numerous subpleural nodules. He was admitted for medical management of NSTEMI, with planned coronary angiogram.

On hospital day 2, the patient underwent left heart cardiac catheterization that revealed non-obstructive coronary artery disease, a very large fistulous connection between the RCA and the SVC [Figure 2].

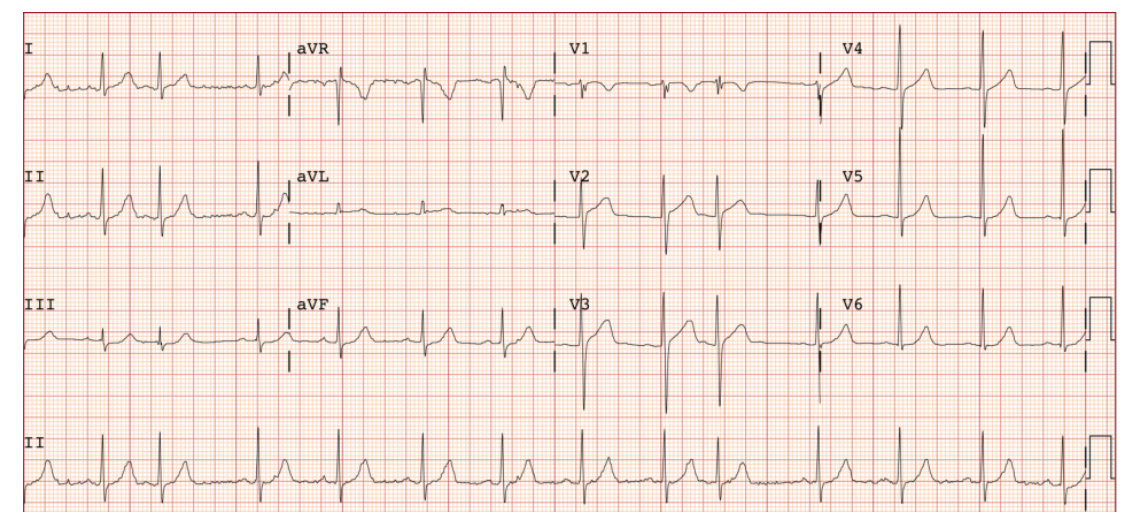

Figure 1. Admission EKG revealing minimal ST depressions in leads II and aVF

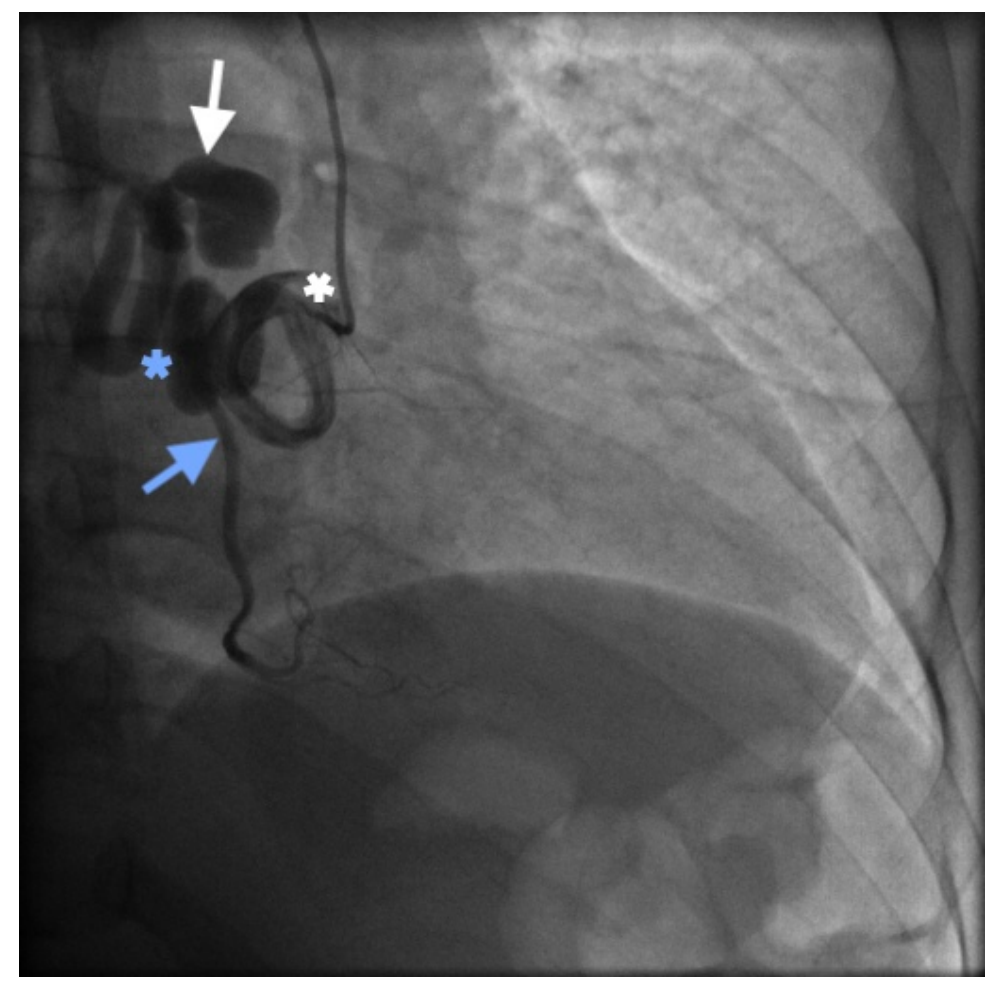

Figure 2. Coronary angiography showing catheter in aorta with catheter tip positioned in the orifice of the RCA (white asterisk). Contrast filling tortuous and convoluted fistula (blue asterisk) with fistula terminating in the SVC (white arrow),. Contrast filling post-fistula origin distal RCA (blue arrow) 


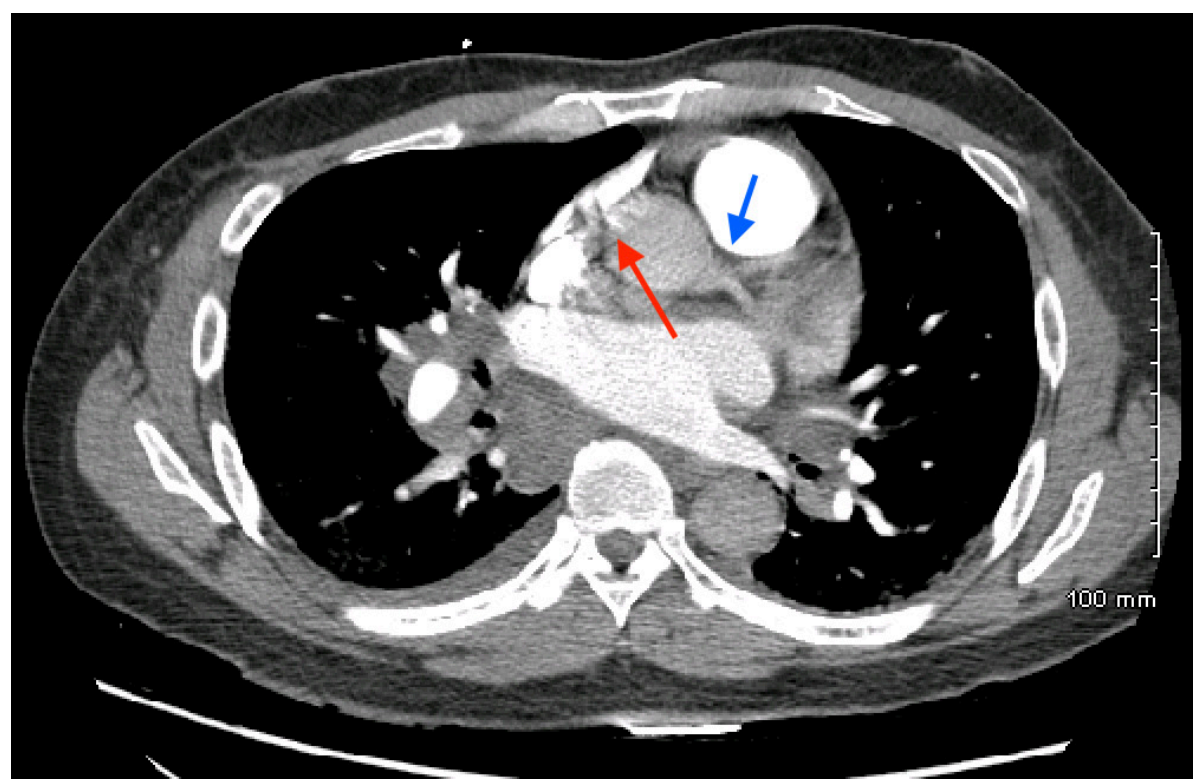

Figure 3. CTA showing RCA-SVC fistulous connections and contrast opacification of the origin of the RCA (red arrow), and the origin of the left coronary artery (blue arrow)

Given extensive lymphadenopathy demonstrated on chest imaging, pulmonology was consulted for further evaluation. Review of CTA images demonstrated fistulous connections revealed on coronary angiogram [Figure 3]. On hospital day 3, the patient underwent bronchoscopy with biopsy from multiple mediastinal and hilar lymph nodes, which later revealed diagnosis of sarcoidosis. On hospital day 5, the patient left against medical advice and was subsequently lost to follow up.

\section{Discussion}

Historically, CAFs are largely asymptomatic till the $5^{\text {th }}$ decade of life, but can manifest in variable symptomatic fashions from angina to arrhythmia, as a murmur on cardiac auscultation, and depending on the degree of shunting, can result in left-sided ischemia with congestive heart failure or right circulatory overload with pulmonary hypertension and cor pulmonale $[3,13]$. Larger CAFs can enlarge overtime, further worsening the shunt and exacerbating symptoms, typically appearing as a systolic crescendo-decrescendo murmur best appreciated in the region of the chest where the fistula terminates [3,14].

If CAF is suspected based on physical exam or incidental finding on chest or cardiac imaging, recommendations on diagnostic imaging and management are included in some major cardiology society guidelines and not explicitly discussed in others. International guidelines on adult congenital heart disease from the Canadian Cardiovascular Society and the European Society of Cardiology do not specifically address CAFs $[15,16]$. Review of guidelines from the American Heart Association and American College of Cardiology highlights important CAF diagnostic and management aspects. While the 2008 guidelines list the preferred CAF diagnostic imaging modalities and surgical and percutaneous methods of fistula closure, the 2018 guidelines instead stress the overall need for a multi-disciplinary medical and surgical team approach and strategy for comprehensive evaluation and care $[17,18]$.
CAF anatomy should be evaluated by echocardiography, magnetic resonance imaging (MRI), CTA, or cardiac catheterization with coronary angiography based on the 2008 recommendations [17]. CAF imaging is crucial to elucidating CAF origin, termination, and complexity, with identification of the artery of origin necessary to evaluate dilation of the feeding artery $[19,20]$. Furthermore, the position of thr CAF origin along the proximal or distal aspect of the coronary artery categorizes the fistula based on the Sakakibara CAF classification system. According to the Sakakibara classifications, CAFs are defined as proximal or distal based on origin artery positioning, as fistulas originating from the proximal third are associated with dilation of the proximal feeding artery and normal distal artery diameter, while fistulas originating from the distal two-thirds tend to cause dilation along the entire length of the feeding artery [19,21]. Given the origin of our patient's CAF as demonstrated on coronary angiogram, this RCA-SVC fistula would be characterized as a proximal CAF. The overall classification of CAF anatomy is a multifaceted process that incorporates the Sakakibara system and additional information on fistula morphology including artery of origin, site of termination, micro- or macro-fistula termination, number of fistulous connections, and diameter [20].

Echocardiographic evaluation, both TTE and TEE, is limited by fields of view and operator dependency, but can delineate $\mathrm{CAF}$ anatomy, and analyze CAF associated hemodynamic and physiologic changes while avoiding radiation exposure $[19,22]$. Use of micro-bubble contrast can enhance echocardiography by more precisely determining the extent and location of the CAF, but has diminished utility in CAFs with distal arterial origins $[19,23,24]$. Current guidelines refer to the use of surveillance echocardiography every 3-5 years for follow up of patients with small and asymptomatic CAF to monitor for fistula progression and terminating chamber enlargement [17]. Magnetic resonance imaging with angiography (MRA) is an evolving noninvasive imaging modality without radiation or contrast exposure for evaluation of CAF [19,25]. However, CAF evaluation 
with MRA is limited by lower spatial resolution and is relatively poorer at visualizing the course of distal coronary arteries $[19,26]$.

While coronary angiography remains the premier diagnostic modality, CTA is considered the superior noninvasive imaging method for CAF $[19,27]$. CTA, with or without multi-detector CTA that enables enhanced visualization of the coronary arteries, yields high spatial and temporal resolution through shorter acquisition time and provides detailed anatomical rendering, further delineated with three-dimensional volume rendering $[19,27,28]$. Unlike the aforementioned techniques that are limited to morphologic evaluation of CAF, coronary angiography ascertains important CAF hemodynamic information that can be further enhanced with use of intravascular ultrasound [27,29]. Ultimately, the recommendation is that all CAFs should undergo coronary angiography for CAF anatomic definition [17].

According to the 2008 ACC/AHA guidelines, CAFs that should be evaluated for closure are large fistulas regardless of symptomology, and small to moderate fistulas that are associated with myocardial ischemia, arrhythmia, endarteritis, unexplained ventricular diastolic or systolic dysfunction, and ventricular dilation [17]. If a CAF is incidentally discovered on imaging and considered to be the etiology of angina, myocardial ischemia can be evaluated with exercise stress testing or myocardial perfusion scanning $[3,27,30]$. CAF size characterization is defined with angiography and in reference to the distal vessel lumen, with a large CAF being greater than $2 \mathrm{x}$ the diameter of the distal vessel, and small to moderate being less than 1-2x the distal vessel diameter [20]. Guideline directed management strategies for CAF closure include transcatheter and surgical techniques that require review of patient and CAF anatomy-centered discussions involving interventional cardiologists, cardiothoracic surgeons and those with training and expertise in congenital heart disease $[17,18]$. The main surgical options employed in CAF management, individually or in combination, include epicardial fistula mobilization with ligation or division, arteriotomy of the dilated proximal coronary artery with suturing close to the fistula origin, and exposure of the fistulous connection and closure by direct suture or autologous pericardial patch [31]. Percutaneous transcatheter CAF closure requires favorable coronary anatomy successful cannulation of the artery of origin, with multiple catheter based closure tools available including occlusion coils, detachable balloons, and deployable stents and balloons [19,31]. Selection of percutaneous or surgical management strategy is largely influenced by the anatomy and physiology of the CAF. Fistulas that are symptomatic, large, high volume of flow, with multiple communications and drainages, and tortuous anatomy are candidates for surgical management [19]. Furthermore, percutaneous transcatheter closure should be considered in CAFs with an origin in the proximal portion of the feeding coronary artery, a non-tortuous anatomy, and in older adult patients with high perioperative risk [19].

In summary, we present the case of an RCA-SVC fistula manifesting as an NSTEMI. While generally remaining asymptomatic throughout adulthood, CAFs can cause angina through myocardial ischemia, but are unlikely to cause infarction with serologic evidence of myocardial ischemia in the absence of intra-fistula thrombosis. Given that CAFs can arise congenitally or from medical conditions and procedures involving the coronary arteries, the prevalence and clinical manifestations of CAFs may rise given the increasing rates of atherosclerotic coronary artery disease, percutaneous coronary intervention, and coronary artery bypass grafting. While the ACC/AHA guidelines from 2008 and 2018 highlight important diagnostic and intervention strategies, further studies and input from international cardiology societies are needed to continue to improve the evaluation and management of CAFs.

\section{Acknowledgements}

This work is supported, in part, by the efforts of Dr. Moro O. Salifu M.D., M.P.H., M.B.A., M.A.C.P., Professor and Chairman of Medicine through NIH Grant number S21MD012474.

\section{References}

[1] Karazisi C, Eriksson P, Dellborg M. Coronary Artery Fistulas: Case Series and Literature Review. Cardiology. 2017; 136(2): 93-101.

[2] Said SAM, Schiphorst RHM, Derksen R, Wagenaar LJ. Coronarycameral Fistulas in Adults: Acquired Types (Second of Two Parts) World J Cardiol. 2013; 5(12): 484-494.

[3] Challoumas D, Pericleous A, Dimitrakaki IA, et al. Coronary Arteriovenous Fistulae: A Review. Int J Angiol. 2014; 23(1): 1-10.

[4] Lim JJ, Jung JI, Lee BY, Lee HG. Prevalence and Types of Coronary Artery Fistulas Detected with Coronary CT Angiography. AJR Am J Roentgenol. 2014; 203(3): W237-243.

[5] Mangukia CV. Coronary Artery Fistula. Ann Thorac Surg. 2012; 93: 2084-2092.

[6] Ghandour A, Rajiah P. Unusual Fistulas and Connections in the Cardiovascular System: A pictorial review. World J Radiol. 2014; 6(5): 169-176.

[7] Pagni S, Austin EH, Abraham JS. Right Coronary Artery to Superior Vena Cava Fistula Presenting with 'steal' Phenomenon. Interactive Cardiovasc Thorac Surg. 2004; 3(4): 573-574.

[8] Dolapoglu A, Ott DA. Giant Right Coronary Artery Aneurysm Associated with a Fistula Draining into the Superior Vena Cava. Texas Heart Inst J. 2016; 43(4): 360-362.

[9] Rigatelli G, Dell'Avvocata F, Van Tan N, et al. Congenital Coronary Artery Anomalies Silent Until Geriatric age: Noninvasive Assessment, Angiography Tips, and Treatment. J Geriatr Cardiol. 2015; 12(1): 66-75.

[10] Ghadri JR, Kazakauskaite E, Braunschweig S, et al. Congenital Coronary Anomalies Detected by Coronary Computed Tomography Compared to Invasive Coronary Angiography. BMC Cardiovasc Disord. 2014; 14: 81.

[11] Smettei OA, Abazid RM. A Rare Cause of Coronary Artery Fistula Presented with Acute Myocardial Infarction. Avicenna J Med. 2015; 5(2): 49-51.

[12] Santos EL, Gouveia MM, Silva RF, et al. Coronary Artery Fistula Causing Acute Myocardial Infarction and Right Ventricle Thrombosis. Cureus. 2018; 10(3): e2314.

[13] Gowda RM, Vasavada BC, Khan IA. Coronary Artery Fistulas: Clinical and Therapeutic Considerations. Int J Cardiol. 2006; 107: 7-10.

[14] Loukas M, St. Germain A, Gabriel A, et al. Coronary Artery Fistula: A Review. Cardiovasc Path. 2015; 24: 141-148.

[15] Silversides CK, Marelli A, Beauchesne, et al. Canadian Cardiovascular Society 2009 Consensus Conference on the Management of Adults with Congenital Heart Disease: Executive Summary. Can J Cardiol. 2010; 26(3): 143-150.

[16] Baumgartner $\mathrm{H}$, Bonhoeffer $\mathrm{P}$, De Groot NM, et al. ESC Guidelines for the Management of Grown-up Congenital Heart 
Disease (New Version 2010). Eur Heart J. 2010; 31(23): 2915-2957.

[17] Warnes CA, Williams RG, Bashore TM, et al. ACC/AHA 2008 Guidelines for the Management of Adults with Congenital Heart Disease: A Report of the American College of Cardiology/American Heart Association Task Force on Practice Guidelines (Writing Committee to Develop Guideliens on the Management of Adults with Congenital Heart Disease). Developed in Collaboration with the American Society of Echocardiography, Heart Rhythm Society, International Society for Adult Congenital Heart Disease, Society for Cardiovascular Angiography and Interventions, and Society of Thoracic Surgeons. JACC. 2008; 52(23): e143-e263.

[18] Stout KK, Daniels CJ, Aboulhosn JA, et al. 2018 AHA/ACC Guideline for the Management of Adults with Congenital Heart Disease: A Report of the American College of Cardiology/American Heart Association Task Force on Clinical Practice Guidelines. Circulation. 2019; 139(14): e698-e800.

[19] Yun G, Nam TH, Chun EJ. Coronary Artery Fistulas: Pathophysiology, Imaging Findings, and Management. Radiographics. 2018; 38(3): 688-703.

[20] Reddy G, Davies JE, Holmes DR, et al. Coronary Artery Fistulae. Circ Cardiovasc Interv. 2015; 8(11): e003062.

[21] Sakakibara S, Yokoyama M, Takao A, et al. Coronary Arteriovenous Fistula. Nine Operated Cases. Am Heart J. 1966; 72(3): 307-314.

[22] Barbosa MM, Katina T, Oliveira HG, et al. Doppler Echocardiographic Features of Coronary Artery Fistula: Report of 8 Cases. J Am Soc Echocardiogr. 1999; 12(2): 149-154.
[23] Goswami NJ, Zabalgoitia M. Localization of a Coronary Artery Fistula Using Contrast Transesophageal Echocardiography. J Am Soc Echocardiogr. 2002; 15(8): 839-840.

[24] Hong SM, Yoon Sj, Rim SJ. Contrast Echo - A Simple Diagnostic Tool for a Coronary Artery Fistula. Korean Circ J. 2012; 42(3): 205-207.

[25] Gharib AM, Ho VB, Rosing DR, et al. Coronary Artery Anomalies and Variants: Technical Feasibility of Assessment with Coronary MR Angiography at 3 T. Radiology. 2008; 247(1): 220-227.

[26] Gerretsen SC, Kooi ME, van Engelshoven JMA, et al. Magnetic Resonance Imaging of the Coronary Arteries. Cardiovase J Afr. 2007; 18(4): 248-259.

[27] Buccheri D, Chirco PR, Geraci S, et al. Coronary Artery Fistulae: Anatomy, Diagnosis, and Management Strategies. Heart Lung Circ. 2018; 27(8): 940-951.

[28] Namgung J, Kim JA. The Prevalence of Coronary Anomalies in a Single Center of Korea: Origination, Course, and Termination Anomalies of Aberrant Coronary Arteries Detected by ECG-gated Cardiac MDCT. BMC Cardiovasc Disord. 2014; 14: 48.

[29] Pepe M, Giardinelli F, Cafaro A, et al. Fractional Flow Reserve and Intravascular Scan as Part of the Coronary Fistulas Diagnostic Process: Future Perspectives. Cardiol J. 2018; 25(3): 414-415.

[30] Lee SK, Jung JI, O JH, et al. Coronary-to-artery Fistula in Adults: Evaluation with Thallium-201 Myocardial Perfusion SPECT. PLoS One. 2017; 12(12): e0189269.

[31] Mangukia CV. Coronary Artery Fistula. Ann Thorac Surg. 2012; 93: 2084-2092.

(C) The Author(s) 2019. This article is an open access article distributed under the terms and conditions of the Creative Commons Attribution (CC BY) license (http://creativecommons.org/licenses/by/4.0/). 